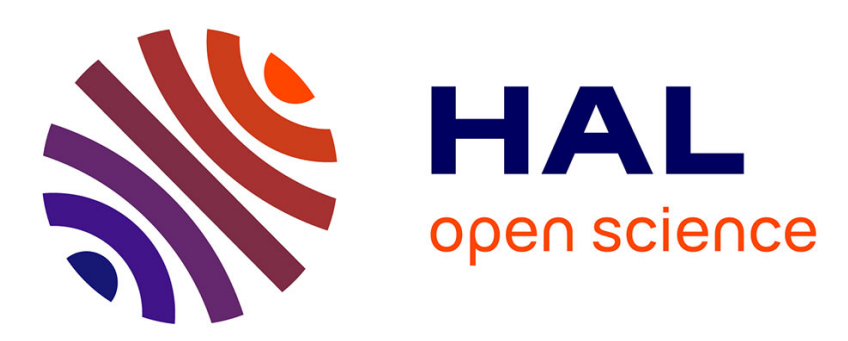

\title{
Etude hydrodynamique de l'expansion d'un plasma avec formation d'anévrisme créé lors de l'interaction entre un faisceau laser et un échantillon de graphite
} M. Ghrab, C. Dumas, C. Prat, M. Autric

\section{- To cite this version:}

M. Ghrab, C. Dumas, C. Prat, M. Autric. Etude hydrodynamique de l'expansion d'un plasma avec formation d'anévrisme créé lors de l'interaction entre un faisceau laser et un échantillon de graphite. Journal de Physique IV Proceedings, 1994, 04 (C4), pp.C4-111-C4-114. 10.1051/jp4:1994423 . jpa00252686

\section{HAL Id: jpa-00252686 \\ https://hal.science/jpa-00252686}

Submitted on 1 Jan 1994

HAL is a multi-disciplinary open access archive for the deposit and dissemination of scientific research documents, whether they are published or not. The documents may come from teaching and research institutions in France or abroad, or from public or private research centers.
L'archive ouverte pluridisciplinaire HAL, est destinée au dépôt et à la diffusion de documents scientifiques de niveau recherche, publiés ou non, émanant des établissements d'enseignement et de recherche français ou étrangers, des laboratoires publics ou privés. 


\title{
Etude hydrodynamique de l'expansion d'un plasma avec formation d'anévrisme créé lors de l'interaction entre un faisceau laser et un échantillon de graphite
}

\author{
M. GHRAB, C. DUMAS, C. PRAT et M. AUTRIC
}

Institut de Mécanique des Fluides, UM 34 du CNRS, Equipe Interaction Laser-Matière, Parc Scientifique et Technologique de Luminy, 13009 Marseille, France

\begin{abstract}
Des expériences de visualisations de jets de plasmas créés par laser à $\mathrm{CO}_{2}$ impulsionnel sur matériaux solides ont été réalisées afin d'étudier le comportement hydrodynamique de ces plasmas créés en atmosphère variable (de $10^{2} \mathrm{~Pa}$ à $10^{5} \mathrm{~Pa}$ ). On $s^{\prime}$ intéressera plus particulièrement dans cette présentation aux plasmas de carbone se propageant dans de l'air synthétique à faible pression. Pour des conditions bien particulières de densité d'énergie et de pression ambiante, le caractère instable d'une onde de souffle a été mis en évidence. Cette instabilité hydrodynamique se caractérise par la formation d'un second front (anévrisme) prenant naissance dans la partie frontale de l'onde de souffle exposée au rayonnement laser.
\end{abstract}

\section{INTRODUCTION}

Lorsqu'un rayonnement laser intense irradie un matériau placé en atmosphère raréfiée, on observe expérimentalement la formation d'une onde de choc sphérique totalement transparente au rayonnement laser. Cette onde de choc, appelée "onde de souffle", résulte d'une compression de l'air ambiant provoquée par l'éjection des particules de la surface du matériau. Le gaz ambiant peut être partiellement ionisé près de la surface par collisions avec ce front de choc. Lorsque le dépôt d'énergie est instantané (pulse court), l'onde de souffle créée est sphérique et se développe proportionnellement à $t^{0,4}$ [1]. Lorsque, comme dans notre cas, l'apport d'énergie dure plusieurs microsecondes, la structure de l'onde est quasisphérique et elle se développe proportionnellement à $\mathrm{t}^{0,6}$ [2].

$\mathrm{Au}$ cours des expériences réalisées sur des échantillons de graphite et pour des pressions ambiantes comprises entre $10^{2} \mathrm{~Pa}$ et $510^{2} \mathrm{~Pa}$ [3], une instabilité hydrodynamique a été observée. Elle se caractérise par la formation d'une seconde onde qui se superpose à l'onde sphérique. Cette onde, appelée "anévrisme", semble prendre naissance dans le front de choc exposé au rayonnement laser ou apparaître dans une direction perpendiculaire à la surface de l'échantillon irradié. Dans ce cas le développement de l'onde initiale suit une loi dévolution temporelle en $\mathrm{t}^{1}$.

Plusieurs mécanismes ont été avancés pour tenter d'expliquer la formation de cet anévrisme [4]:

- L'échauffement du canal irradié par le faisceau laser, permet à cette région du milieu ambiant de se propager comme une onde de choc cylindrique et de former par la suite une zone à faible densité. L'onde de souffle sphérique peut alors se propager plus rapidement dans cette zone, et former ainsi l'anévrisme. Ce chauffage laser peut s'expliquer totalement par le processus d'absorption par bremsstrahlung inverse.

- Un jet de matériaux vaporisé peut être à l'origine de l'anévrisme. Comme le front de l'onde de souffle est freiné au fur et à mesure de sa propagation dans le milieu ambiant, le jet localisé peut se détacher de l'onde pour former un anévrisme. Ce mécanisme peut être effectif si l'anévrisme est orienté normalement à la surface irradiée.

- Un autre mécanisme réside dans l'onde elle-même. La décélération du front de souffle est grande. L'instabilité hydrodynamique du front ainsi créée peut provoquer la formation d'un anévrisme [4]. 
- Le quatrième mécanisme proposé dépend de l'évaporation thermique locale à l'intérieur du front de l'onde formant ainsi une région préférentielle d'ablation accélérée. Un front de conduction thermique est évoqué entre le front de l'onde de souffle et le plasma chaud. Les champs magnétiques auto-générés [4], qui sont azimutaux autour de l'axe central et intenses au delà, fournissent une isolation thermique sur la face du front de l'onde mis à part dans la région de l'axe où les champs sont nuls. Ainsi, l'intérieur du front de l'onde interagit plus avec la surface et projette un anévrisme à l'extérieur de la partie sphérique.

\section{CONDITIONS EXPERIMENTALES ET MOYENS DE MESURES}

Les expériences réalisées au cours de cette étude ont été effectuées au moyen d'un laser à $\mathrm{CO}_{2}$ impulsionnel pouvant délivrer jusqu'à 1000 Joules en une impulsion de $10 \mu \mathrm{S}$.

Au cours des essais, la durée de l'impulsion a été limitée à $4 \mu \mathrm{s}$. La densité de puissance incidente moyenne sur l'échantillon a été fixé à $85 \mathrm{MW} / \mathrm{cm}^{2}$.

Le montage expérimental réalisé à cet effet est basé sur le principe de l'interféromètre de Mach-Zehnder (Figure 1). La source lumineuse servant pour la formation du système de franges est un laser argon multiraies d'une puissance de $4 \mathrm{~W}$ et dont nous utilisons la raie la plus intense de longueur d'onde $5145 \AA$ A.

Un système composé d'un miroir plan (M6) et un miroir sphérique (M9) permet de former l'image du plasma, avec un grandissement présélectionné, sur le diaphragme d'entrée d'une caméra ultra-rapide à convertisseur d'images (Imacon 790).

\section{RESULTATS EXPERIMENTAUX}

Les visualisations réalisées à la pression de $10^{2} \mathrm{~Pa}$, que ce soit en ombroscopie ou en interférométrie, montrent la formation d'une onde de choc qui prend naissance dès le début de l'initiation du plasma ( $\sim 70$ ns après le début de l'impulsion laser, ce qui correspond au pic de l'impulsion).

Environ 130 ns après l'initiation du plasma, on assiste à la formation de l'anévrisme, qui prend naissance sur la partie frontale de (P2) irradiée par la faisceau laser (Figure 2). Cette zone préchauffée et préionisée est favorable à linitiation du processus d'ionisation en cascade conduisant à la formation de l'anévrisme qui n'est autre qu'une onde d'absorption.

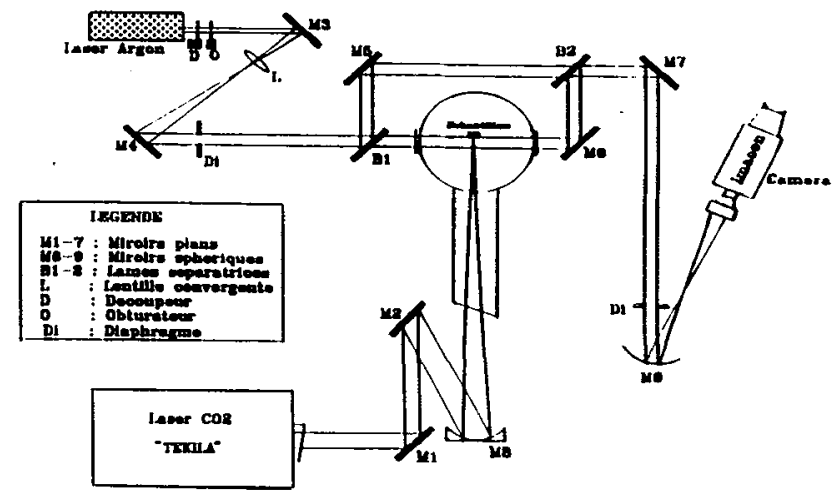

Fig. 1 : Dispositif expérimental

A partir de plusieurs essais réalisés dans les même conditions ( $\mathrm{P}=10^{2} \mathrm{~Pa}$ ), on a constaté que l'anévrisme se forme toujours dès que l'épaisseur entre les deux fronts (P2-P3) dépasse $0.3 \mathrm{~cm}$ Ceci peut être expliqué par le fait que l'onde d'absorption ne peut être initiée que si la zone considérée est suffisamment dense, et par suite épaisse, pour pouvoir absorber une grande partie de l'énergie incidente. Ce qui constitue une étape primordiale pour tout processus d'ionisation en cascade. 
L'expansion axiale (P2) ainsi que l'expansion radiale (R) du premier front sont proportionnelles à (t) (Figure 3), ce qui implique qu'il ne s'agit pas d'une onde de souffle sphérique qu'on peut traiter à part sans tenir compte de l'effet induit par l'onde d'absorption (ou "anévrisme"). En effet, celle-ci engendre un écoulement de l'air choqué dans la direction de la surface de l'échantillon [5]. Cet écoulement induit a pour conséquence l'augmentation de la vitesse radiale de l'onde, qui se traduit par une évolution du rayon de l'onde proportionnelle à $\left(\mathrm{t}^{1}\right)$, et non à $\left(\mathrm{t}^{0.6}\right)$ ou $\left(\mathrm{t}^{0.4}\right)$ prévue par la théorie de l'onde de souffle.

Pour vérifier l'hypothèse de l'existence d'une onde d'absorption entretenue par laser, une série d'essais sur échantillon incliné de $20^{\circ}$ par rapport à l'axe du faisceau incident a été
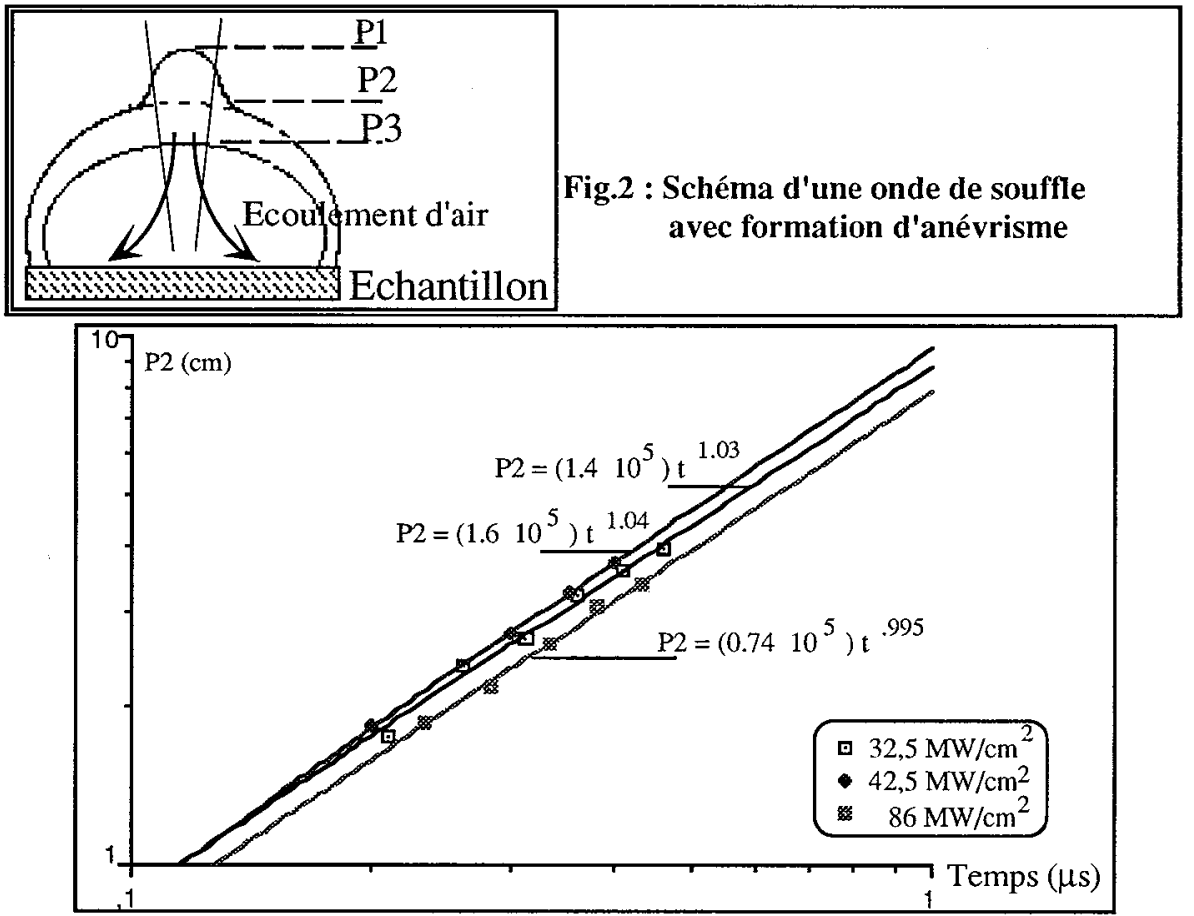

Fig. 3 : Evolution du deuxième front de l'écoulement à $P=10^{2} \mathrm{~Pa}$

réalisée (Figure 4). Ceci a permis de séparer l'onde d'absorption entretenue par laser qui se propage toujours dans la direction du faisceau laser, de l'onde de souffle transparente, qui, elle, se propage suivant une direction perpendiculaire à la surface irradiée [6].

Les essais réalisés confirment bien l'hypothèse avancée plus haut concernant l'existence d'un front d'absorption entretenu par laser. En effet, l'anévrisme prend naissance aux alentours de l'axe du faisceau laser et se propage dans sa direction d'une façon identique à celle observée dans le cas des essais avec surface perpendiculaire au faisceau incident.

Par contre, la propagation de l'onde de souffle n'est pas identique à celle observée dans le cas d'une surface perpendiculaire. Ceci a été mis en évidence par la mesure des positions (Z1) et (R) (Figure 5) pour les différents instants de prise de vues photographiques, et qui démontrent que leurs évolutions sont respectivement proportionnelles à $\left(t^{0.74}\right)$ et $\left(\mathrm{t}^{0.55}\right)$ :

$$
\begin{aligned}
& Z 1 \sim \text { at } \\
& R \sim \text { b t }
\end{aligned}
$$

Z1, R en mètre

$t$ en seconde

L'inclinaison de la surface a permis de réduire la contribution de l'onde d'absorption sur l'évolution radiale de l'onde de souffle, qui, dans la configuration actuelle, suit une loi 
d'évolution en $\left(t^{0.55}\right)$. Cette évolution est très proche de celle théorique, décrivant une onde de souffle sphérique avec un dépôt d'énergie variable.
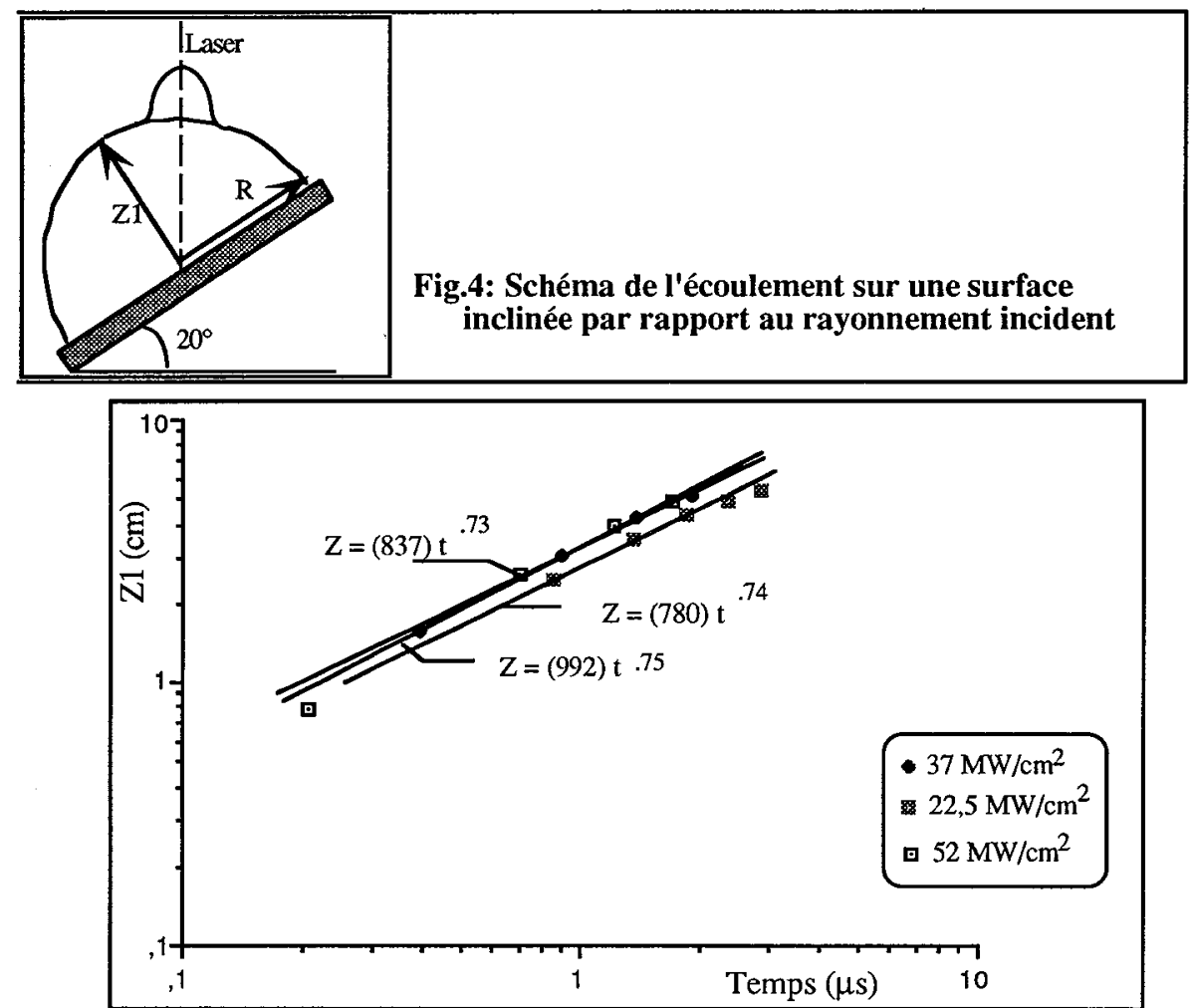

Fig.5 : Evolution perpendiculaire de l'onde de choc sur surface inclinée $\left(P=10^{2} \mathrm{~Pa}\right)$

Contrairement à l'évolution radiale, la propagation de l'onde de souffle dans la direction normale à la surface est influencée par la présence de l'anévrisme qui, malgré l'absorption d'une grande partie de l'énergie incidente, se propage avec une vitesse proche de celle de l'onde de souffle (dans la direction normale à la surface). Ceci suppose l'existence d'un important transfert d'énergie entre ces deux fronts, surtout aux abords de l'anévrisme et qui se traduit par une vitesse normale de l'onde $(Z 1 \sim$ a t 0.74 ) plus importante que la vitesse radiale qui reste peu influencée par la présence de l'anévrisme.

\section{REFERENCES}

[1] : L. SEDOV, "Similarity and dimensional methods in mechanics", Academic Press, New york (1959).

[2] : E. K. DABORA, "variable energy blast waves", A.I.A.A. Joumal, Vol.10, Octobre (1972)

[3] : M. GHRAB, "Caractérisation de jets de plasmas crés par laser à $\mathrm{CO}_{2}$ impulsionnel sur matériaux solides", Thèse de doctorat de l'université d'Aix-Marseille II, No 207-93-43, Octobre (1993).

[4] : J. A. STAMPER, "Aneurisms in laser-driven blast waves", Phys. Fluids, 31 (11), 3353-3361, (1988).

[5] : A. N. PIRRI, R. G. ROOT \& al, "Plasma energy transfert to metal surfaces irradiated by pulsed lasers", A.I.A.A.Joumal, 16 (12), 1296-1304, (1978)

[6] : W. E. MAHER, R. B. HALL, "Experimental study of effects from two laser pulses", J. Appl. Physics, Vol. 47, N6, 2486-2493, (1976) 